\title{
Osteoadherin serves roles in the regulation of apoptosis and growth in MC3T3-E1 osteoblast cells
}

\author{
ERI HAMAYA $^{1,2}$, TOSHIAKI FUJISAWA ${ }^{1}$ and MASATO TAMURA ${ }^{2}$ \\ Departments of ${ }^{1}$ Dental Anesthesiology and ${ }^{2}$ Biochemistry and Molecular Biology, Faculty of Dental Medicine and \\ Graduate School of Dental Medicine, Hokkaido University, Sapporo, Hokkaido 060-8586, Japan
}

Received May 24, 2019; Accepted September 19, 2019

DOI: $10.3892 /$ ijmm.2019.4376

\begin{abstract}
Small leucine-rich proteoglycans (SLRPs) are a class of proteoglycans that are characterized by small protein cores and structures of leucine-rich repeats. SLRPs are expressed in most extracellular matrices and share numerous biological functions that are associated with binding of collagens and cell surface receptors. Osteoadherin (also termed osteomodulin) is encoded by the Omd gene and is a keratan sulfate proteoglycan of the class II subfamily of SLRPs. Osteoadherin is highly expressed in mineralized tissues, including bone and dentin; however, it's precise roles remain unknown. The present study determined the $O m d$ expression levels and investigated the effects of over- and under-expression of osteoadherin in osteoblastic cells. Omd mRNA expression increased with osteoblast differentiation in MC3T3-E1 cells. In $\mathrm{C} 2 \mathrm{C} 12$ cells, Omd mRNA expression was induced by bone morphogenetic protein (BMP)2. Reporter assays similarly demonstrated activation of the $O m d$ gene promoter following co-transfection with Smad1 and Smad4, which are intracellular signaling molecules of the BMP2 signaling pathway. Overexpression of $\mathrm{Omd}$ increased the viability and decreased caspase 3/7 activity in MC3T3-E1 cells. By contrast, following transfection with small interfering RNA for $O m d$, viable cell numbers were decreased and caspase 3/7 activity was increased. Furthermore, overexpression of $\mathrm{Omd}$ reduced the expression of $\mathrm{CCN}$ family 2 in these cells. These results demonstrate that $\mathrm{Omd}$ expression is regulated during osteoblast differentiation, and that the protein product osteoadherin serves roles in the apoptosis and growth of osteoblast cells.
\end{abstract}

Correspondence to: Professor Masato Tamura, Department of Biochemistry and Molecular Biology, Faculty of Dental Medicine and Graduate School of Dental Medicine, Hokkaido University, North 13, West 7, Sapporo, Hokkaido 060-8586, Japan

E-mail: mtamura@den.hokudai.ac.jp

Key words: osteoadherin, Omd, small leucine-rich proteoglycan, osteoblast, apoptosis

\section{Introduction}

Extracellular matrices (ECMs) provide cell microenvironments with several secreted molecules, such as collagens, glycoproteins, glycosaminoglycans (GAGs) and proteoglycans. Proteoglycans regulate cell behaviors by binding GAG chains or through direct protein-protein interactions via the core protein (1). The structure of small leucine-rich proteoglycans (SLRPs) consists of a small protein core with various GAG side chains, and SLRPs are grouped into five classes based on common structural properties $(1,2)$. Osteoadherin, also termed osteomodulin (the $\mathrm{Omd}$ gene product), was originally isolated from guanidine extracts of bovine bone, and is a 47-kDa keratan sulfate proteoglycan of the class II subfamily of SLRPs $(2,3)$. Several SLRPs are ECM components; however, osteoadherin is the only member that is restricted to mineralized tissues, such as bones and teeth (4-6). In bone tissues, $O m d$ is expressed by fetal and adult osteoblasts, and its product osteoadherin can reach up to $400 \mu \mathrm{g} / \mathrm{g}$ wet weight in bovine bone (3).

As with numerous SLRPs, osteoadherin binds a number of ECM components; in particular, it binds fibrillar collagens to stabilize fibrillar tissue frameworks $(7,8)$. Leucine-rich repeat (LRR) motifs comprise 6-10 repeats of $\sim 20-29$ amino acids with conserved leucine spacing, and are folded into structures with one $\beta$-sheet and one $\alpha$-helix. LRRs participate in a wide range of biological processes, and interact directly with collagen, heparin-binding proteins and cell surface receptors to block ligand binding (9). Osteoadherin contains six closely spaced tyrosine sulfate residues in its $\mathrm{N}$-terminal region and two in its C-terminal region $(9,10)$. Tyrosine sulfate domains bind heparin-binding proteins, such as basic growth factor-2 and thrombospondin I (10). Osteoadherin also contains large numbers of acidic amino acid residues in its $\mathrm{N}$-terminal region (3), which may bind to hydroxyapatite in calcified tissues and basic cluster motifs of heparin-binding proteins and growth factors $(9,11)$. SLRPs also carry GAG chains that are covalently attached to the core protein, and can modulate biological functions depending on the nature of GAG chains. Through these interactions, SLRPs are involved in the initial triggering of multiple cellular responses in various tissues (2). However, it is unclear whether osteoadherin in bone tissues serves roles in the regulation of osteoblast growth, apoptosis, and differentiation. 
Bone morphogenetic proteins (BMPs) regulate differentiation and proliferation in various cell types, including osteoblasts (12). BMP2 reportedly triggers osteoblast differentiation and upregulates several genes that encode osteoblast phenotype-related proteins in vitro (13). In the intracellular BMP2 signaling pathway, Smad1 is phosphorylated directly by type I receptors for BMP2 and is transported into the nucleus in complexes with Smad4 (14). This complex interacts with the regulatory elements of target genes and regulates their expression. To date, little is understood of how $\mathrm{Omd}$ expression is regulated in response to BMP2 during osteoblast differentiation.

Apoptosis can be initiated by various stimuli and is directed by a family of cysteine proteases known as caspases. At the molecular level, caspase activation plays a central role in the execution of apoptosis. To the best of our knowledge, so far, 14 mammalian caspases have been identified, three of which (caspase-3, -6 and -7) are the effector caspases that coordinate the execution phase of apoptosis by degrading multiple substrates, including the structural and regulatory proteins in the cell nucleus, cytoplasm and cytoskeleton (15).

The present study determined the Omd expression levels during osteoblast differentiation and investigated the effects of overexpression and knockdown of osteoadherin in osteoblast cells. Overexpression of $\mathrm{Omd}$ increased the numbers of viable cells and decreased the activity of caspase 3/7 in MC3T3-E1 cells. By contrast, knockdown of $O m d$ decreased viable cell numbers and increased caspase 3/7 activity in these cells. Omd overexpression also reduced the expression of CCN family 2 (CCN2). Thus, it can be concluded that osteoadherin regulates apoptosis and growth in osteoblast cells.

\section{Materials and methods}

Reagents. Recombinant human BMP2 was kindly supplied by Astellas Pharma Ltd. Dexamethasone and 3-isobutyl-1-methylxanthine (IBMX) were purchased from Sigma-Aldrich; Merck KGaA.

Cell cultures. MC3T3-E1 mouse osteoblast cells and C2C12 myoblast cells were obtained from RIKEN BioResource Center and were cultured in $\alpha$-minimal essential medium ( $\alpha$-MEM; Sigma-Aldrich; Merck KGaA) containing $100 \mu \mathrm{g} / \mathrm{ml}$ kanamycin (Meiji Seika Kaisha, Ltd.) and 10\% fetal bovine serum (FBS; Sigma-Aldrich; Merck KGaA) at $37^{\circ} \mathrm{C}$ under a humidified atmosphere containing $5 \% \mathrm{CO}_{2}$ as described previously (16). MC3T3-E1 cells were cultured and differentiated in $\alpha$-MEM containing $10 \%$ FBS, $10 \mathrm{mM} \beta$-glycerophosphate (Tokyo Chemical Industry Co., Ltd.) and $50 \mu \mathrm{g} / \mathrm{ml}$ ascorbic acid (Wako Pure Chemical Industries, Ltd.) for 1-3 weeks. Pre-adipocyte 3T3-L1 cells were purchased from DS Pharma Biomedical Co., Ltd. and grown in Dulbecco's modified Eagle's medium (DMEM; Sigma-Aldrich; Merck KGaA) supplemented with $10 \% \mathrm{FBS}$ at $37^{\circ} \mathrm{C}$ until confluence. At 2 days after confluence (day 0 ), differentiation was induced in adipocyte cultures by adding $500 \mu \mathrm{M}$ IBMX, $10 \mu \mathrm{g} / \mathrm{ml}$ insulin (Cell Science and Technology Institute, Inc.) and $1 \mu \mathrm{M}$ dexamethasone to the basal medium. On day 3 , media were replaced with adipogenic medium comprising DMEM supplemented with $10 \% \mathrm{FBS}$ and $10 \mu \mathrm{g} / \mathrm{ml}$ insulin, which was changed every
2 days thereafter until analysis. $\mathrm{C} 2 \mathrm{C} 12$ cells were seeded into culture dishes, and then cultured in media containing 50 or $200 \mathrm{ng} / \mathrm{ml}$ of BMP2 for 6-24 h. Cells of the mouse stromal cell line ST2 (RIKEN BioResource Center) were obtained and cultured as described previously (17). Similarly, bEnd.3 mouse vascular endothelial cells (American Type Culture Collection) (18) were cultured in DMEM supplemented with $10 \%$ FBS as described previously (19) and culture media were changed every 3-4 days.

Reverse transcription (RT)-semi-quantitative PCR (qPCR). Total RNA was extracted from cells using ISOGEN (Nippon Gene Co., Ltd.) and complementary DNA was synthesized with the Omniscript RT kit (Qiagen $\mathrm{GmbH}$ ) using an oligo(dT) primer $(1 \mu \mathrm{M})$ at $37^{\circ} \mathrm{C}$ for $30 \mathrm{~min}$. semi-qPCR analyses were performed with Taq DNA polymerase (Qiagen $\mathrm{GmbH}$ ) as described previously (20). The specific primer sequences for each gene are listed in Table I. Each reaction consisted of an initial denaturation at $94^{\circ} \mathrm{C}$ for $3 \mathrm{~min}$ followed by three-step cycling: Denaturation at $94^{\circ} \mathrm{C}$ for $30 \mathrm{sec}$, annealing at a temperature optimized for each primer pair for $30 \mathrm{sec}$, and extension at $72^{\circ} \mathrm{C}$ for $40 \mathrm{sec}$. After the requisite number of cycles (25-35 cycles), reactions underwent a final extension at $72^{\circ} \mathrm{C}$ for $5 \mathrm{~min}$. To accommodate the differences in RNA quantities, expression levels were normalized to those of the housekeeping gene GAPDH. Amplification products were separated using electrophoresis on $2 \%$ agarose gels and visualized by SYBR Green staining followed by UV light illumination. The relative intensity of the gel bands was measured using ImageJ software (version 1.52a; National Institutes of Health), and results were normalized to the GAPDH mRNA level.

$R T-q P C R$. Total RNA was extracted from cells using ISOGEN (Nippon Gene Co., Ltd.) and complementary DNA was synthesized with High-Capacity cDNA Reverse Transcription kit (Applied Biosystems; Thermo Fisher Scientific, Inc.) at $37^{\circ} \mathrm{C}$ for $60 \mathrm{~min}$. RT-qPCR analyses were performed using the assay-on-demand TaqMan probes (Mm00449589_m1 for Omd, Mm00650798g1 for Npylr, Mm00475834_m1 for Alp, Mm03413826_mH for Bglap, Mm01192932_g1 for Ccn2, and Mm99999915_g1 for GAPDH; Applied Biosystems; Thermo Fisher Scientific, Inc.) and the StepOne ${ }^{\circledR}$ real time PCR system, according to the manufacturer's protocol, as described previously (21). The qPCR thermocycling conditions included an initial denaturation of $95^{\circ} \mathrm{C}$ for $10 \mathrm{~min}$ followed by 40 cycles of $95^{\circ} \mathrm{C}$ for $15 \mathrm{sec}$ and $60^{\circ} \mathrm{C}$ for $1 \mathrm{~min}$. Relative gene expression levels were quantified using the comparative $2^{-\Delta \Delta \mathrm{Cq}}$ method (22) using GAPDH expression as an endogenous control.

Western blot analysis. MC3T3-E1 cells were transfected according to the experimental conditions and then collected at specific time points, washed in ice-cold PBS, and suspended in CelLytic-M Mammalian cell lysis/extraction reagent (Sigma-Aldrich; Merck KGaA) plus a protease inhibitor (Complete mini; Roche Diagnostics). Whole-cell extracts were quantified using a Protein assay reagent (Bio-Rad Laboratories, Inc.) and $\sim 30 \mu \mathrm{g}$ protein/lane was separated using $10 \%$ SDS-PAGE followed by transfer to a polyvinylidene fluoride membrane (EMD Millipore). Blocking was performed 
Table I. Primers used for reverse transcription-semi-quantitative PCR.

\begin{tabular}{lllc}
\hline Specificity & \multicolumn{1}{c}{ Forward sequence $\left(5^{\prime}-3^{\prime}\right)$} & \multicolumn{1}{c}{ Reverse sequence (5'-3') } & Predicted size, bp \\
\hline Omd & ATGGTGTATTCGCTAAACTTTCAA & AAGATGATTATAGCAGAGGTCAAG & 194 \\
Bgn & TGCCACCGCCATTGTTCCATC & GTTTGTGGAGGGACTGGCTAGA & 486 \\
D $n$ & ATGGCAGTCTGGCCAATGTT & GCCGTCTGAGGGTTACTTGT & 308 \\
Fmod & CATGATCTGCACCCCTTCTT & AATGCAGAGGAAGCCAGGTC & 300 \\
Lum & CCTGAGGAATAACCAAATCGAC & AGACCAGCAGGCAGCTTGCTCA & 393 \\
GAPDH & TCCACCACCCTGTTGCTGTA & ACCACAGTCCATGCCATCAC & 452 \\
\hline
\end{tabular}

Omd, osteoadherin; Dcn, decorin; Bgn, biglycan; Fmod, fibromodulin; Lum, lumican.

with $0.3 \%$ skim milk (BD Biosciences) dissolved in TBS with $0.05 \%$ Tween-20 (TBST) for $1 \mathrm{~h}$ at room temperature. The membrane was probed with antibodies against osteoadherin (rabbit polyclonal anti-human polyclonal antibody; 1:2,000; cat. no. NBP2-19626; Novus Biologicals, LLC) or $\beta$-actin (rabbit polyclonal antibody; 1:2,000; cat. no. 20536-1-AP, ProteinTech Group, Inc.) for $1 \mathrm{~h}$ at room temperature. The membrane was subsequently washed three times and incubated with anti-mouse horseradish peroxidase-linked antibody (1:20,000; cat. no. NA934; GE Healthcare Life Sciences) in TBST for $1 \mathrm{~h}$ at room temperature. Following three additional washes, chemiluminescence detection was performed with ECL Prime Western Blotting Detection Reagent (GE Healthcare Life Sciences) and a LAS 1000 image analyzer, according to the manufacturer's instructions.

Reporter constructs and assays of luciferase activity. Luciferase reporter plasmids for the murine Omd promoter were generated as follows. The 1774-bp Omd promoter fragment $(-1,609$ to +165$)$ (chromosome 13; MGI: 1350918) was isolated from mouse genomic DNA using PCR and was subcloned into the pGL4.12 vector (Promega Corporation) to generate the luciferase reporter plasmid pOmd-luc. The nucleotide sequences of promoter regions were verified by sequencing. The Smad expression plasmids encoding wild-type Smad4 and constitutively active Smad1 (DVD) were provided by Dr Katagiri (Saitama Medical University, Saitama, Japan) (23). For the reporter assay, C2C12 cells were plated $24 \mathrm{~h}$ prior to transfection at a density of $1 \times 10^{5}$ cells/well (24-well plate) and cultured in $\alpha$-MEM supplemented with $10 \%$ FBS. Transfection of plasmid DNA into cells was performed using Lipofectamine $^{\circledR} 2000$ (Invitrogen; Thermo Fisher Scientific, Inc.). Luciferase reporter assays were performed as described previously (20). Briefly, cells were lysed with a PicaGene cell culture lysis reagent Luc (Wako Pure Chemical Industries, Ltd.; cat. no. 300-04351) and firefly luciferase activities were measured using a Mini Lumat LB 9506 luminometer (Berthold Technologies). Luciferase activity was normalized by comparison with $\beta$-galactosidase activity and fold-changes in the luciferase activities of transfected cells were calculated on the basis of those in control MC3T3-E1 cells that were transfected with pcDNA3.

Transfection of small interfering RNAs (siRNAs). MC3T3-E1 cells were transfected with $2.5 \mathrm{nM}$ Silencer Select pre-designed siRNAs for osteoadherin (siOmd; Thermo Fisher Scientific Inc.; cat. nos. s77492 and s77494), the Y1 receptor (siY1; Thermo Fisher Scientific, Inc.; cat. no. s70765) or Silencer negative control siRNA no. 1 (siCont; Thermo Fisher Scientific, Inc.; cat. no. 4390843) using Lipofectamine RNAiMAX (Invitrogen; Thermo Fisher Scientific, Inc.) as previously described (16). Total RNA was extracted after 2 or 6 days for the determination of mRNA level. Total protein was extracted after 2 days for western blot analysis.

Generation of plasmid construct and overexpression of Omd. Omd overexpression plasmid was generated as follows and was subsequently designated pOmd. Total RNA was extracted from MC3T3-E1 cells using ISOGEN (Nippon Gene Co., Ltd.). Mouse Omd complementary DNA was reverse-transcribed from RNA using Omniscript RT kit (Qiagen), and then amplified using primers that flank the mouse Omd open reading frame (forward, 5'-CCAGCCCGAGGACAAGAAAA-3' and reverse, 5'-GGCTTTATGGAGGCATAAATGTGT-3') and PrimeSTAR Max DNA polymerase (Clontech Laboratories, Inc.), according to the manufacturer's instructions. PCR products were electrophoresed on $1 \%$ agarose gels and were purified and subcloned into KpnI and $\mathrm{XbaI}$ restriction sites of the pcDNA3 vector (Invitrogen; Thermo Fisher Scientific, Inc.) using In-Fusion HD cloning kit (Clontech Laboratories, Inc.; cat. no. 639648), according to the manufacturer's protocol. Individual clones of transformed Escherichia coli were isolated from agar plates and nucleotide sequences of each plasmid were confirmed by DNA sequencing. MC3T3-E1 cells were transfected in 24-well plates with $0.1 \mu \mathrm{g}$ pOmd or empty vector (pcDNA3) using Lipofectamine 2000 (Invitrogen; Thermo Fisher Scientific, Inc.) according to the manufacturer's protocol.

Alkaline phosphatase (ALP) staining analyses. MC3T3-E1 cells were plated onto 24 -well plates at a density of $1.7 \times 10^{4} / \mathrm{ml}$. Following $24 \mathrm{~h}$, cells were transfected in 24-well plates with $0.1 \mu \mathrm{g}$ pOmd or pcDNA3 and were cultured in $\alpha$-MEM for 14 days. ALP staining was performed as previously described (20). Briefly, cells were rinsed in PBS, fixed in $10 \%$ formalin at room temperature for $30 \mathrm{~min}$, rinsed again in PBS, and then incubated at room temperature for $1 \mathrm{~h}$ in $300 \mu \mathrm{l}$ aliquots of solution containing $0.15 \mathrm{mg} / \mathrm{ml}$ 5-bromo-4 chloro-3-indolylphosphate, $0.3 \mathrm{mg} / \mathrm{ml}$ nitro-blue tetrazolium (Wako Pure Chemical Industries, Ltd.), $0.1 \mathrm{M}$ Tris- $\mathrm{HCl}$ 
(pH 9.0), $0.01 \mathrm{~N} \mathrm{NaOH}$ and $0.05 \mathrm{mM} \mathrm{MgCl}_{2}$. Images were captured using an iPhone7 smartphone (Apple Corporation). The same settings were applied to all experimental samples.

Quantitation of cell viability. Cell counting kit-8 (Dojindo Molecular Technologies, Inc.; cat no. CK04-01) were used to evaluate cell viability. In these experiments, $10-\mu 1$ aliquots of the WST-8 substrate [5-mM 2-(2-methoxy-4-nitrophenyl)-3-(4-nitr ophenyl)-5-(2,4-disulfophenyl)-2H-tetrazolium, monosodium salt] were added to each well. After incubation for $1 \mathrm{~h}$ at $37^{\circ} \mathrm{C}$, optical densities were measured at a wavelength of $450 \mathrm{~nm}$ using a microplate absorbance reader. Data are expressed as fold changes of transfected samples relative to cell numbers in samples transfected with control siRNA or pcDNA3.

Measurements of caspase 3/7 activity. The enzyme activities of caspase 3/7 were determined using a caspase colorimetric assay (Caspase-Glo 3/7 assay system; Promega Corporation) as described previously (21). Briefly, for each reaction, cells were lysed and incubated with luminogenic substrates containing the Asp-Glu-Val-Asp sequence, which is cleaved by activated caspase 3/7. Following incubation at room temperature for $1 \mathrm{~h}$, luminescence was quantified using a Mini Lumat LB 9506 luminometer (Berthold Technologies). Fold changes in caspase 3/7 activity in transfected cells were calculated on the basis of those in control siRNA or pcDNA3 transfected MC3T3-E1 cells.

Statistical analysis. Data are expressed as the mean \pm standard deviation of three to six independent experiments. Comparison of two groups was performed with a Student's t-test using Microsoft Excel version 2016 (Microsoft Corporation). Comparisons of multiple groups were performed using one-way analysis of variance using Microsoft Excel version 2016 (Microsoft Corporation) followed by Dunnett's multiple comparison test using software provided by Osaka University, Osaka, Japan (http://www.gen-info.osaka-u. ac.jp/MEPHAS/dunnett-e.html). $\mathrm{P}<0.05$ was considered to indicate a statistically significant difference.

\section{Results}

SLRP mRNA expression levels in cultured cells. To evaluate the roles of osteoadherin in mesenchymal cells, Omd mRNA expression levels were determined in various mesenchymal cell cultures and compared with those of other SLRPs, including biglycan, decorin, fibromodulin, and lumican, using RT-semi-qPCR analyses. Omd mRNA was expressed in MC3T3-E1 cells (osteoblasts) but not in C2C12 myoblasts, adipocyte differentiated 3T3-L1 cells, ST2 bone marrow stromal cells or bEnd.3 endothelial cells (Fig. 1A). By contrast, biglycan (Bgn), decorin (Dcn) and fibromodulin (Fmod) mRNA expression was detected in MC3T3-E1, C2C12 and 3T3-L1 cells. Meanwhile, lumican ( $\mathrm{Lum}$ ) mRNA was expressed only in MC3T3-E1 and 3T3-L1 cells. Analysis of the Omd and $B g n$ mRNA/GAPDH mRNA ratio in various mesenchymal cell cultures is presented in Fig. 1B. These data indicate that $O m d$ expression varies during osteoblast differentiation. To determine whether Omd expression reflects the stages of osteoblast differentiation, MC3T3-E1 cells were cultured in differentiation medium for 1-3 weeks and expression levels of Omd mRNA were determined using RT-qPCR. Omd mRNA expression was significantly increased from 1 week and increased further over the culture period of 3 weeks in differentiating osteoblast cells (Fig. 1C). Protein levels were also assayed using western blot analysis and were identified to be increased during in vitro osteoblastic differentiation in MC3T3-E1 cells (Fig. 1D).

BMP2 induces Omd expression in C2C12 cells and regulates Omd promoter activity via Smad signaling. To further investigate the roles of osteoadherin in osteoblast differentiation, experiments were performed with $\mathrm{C} 2 \mathrm{C} 12$ cells, which are well-characterized model cells that differentiate into myotubes or osteoblasts by the presence of BMP2 (13). In C2C12 cells, Omd mRNA expression was not detected using RT-semi-qPCR analyses (Fig. 1A). However, in subsequent RT-qPCR analyses, Omd mRNA expression was increased $>10$-fold after treatment with $50 \mathrm{ng} / \mathrm{ml} \mathrm{BMP2}$. A higher BMP 2 concentration increased the expression of $O m d$ mRNA to $>30$-fold at $200 \mathrm{ng} / \mathrm{ml}$ (Fig. 2A). In time course analyses of BMP2-induced Omd mRNA expression, Omd mRNA expression increased after $6 \mathrm{~h}$, and a significant time-dependent increase in mRNA level was observed at $24 \mathrm{~h}$ (Fig. 2B). These findings indicate that BMP2 induces $O m d$ expression in these cells.

To elucidate the mechanisms by which BMP2 signaling activates $O m d$ transcription, the present study cloned an $\sim 1.7 \mathrm{~kb}$-pair mouse genomic DNA fragment corresponding to the 5'-flanking promoter region of the mouse Omd gene. To determine responsiveness to BMP2 signaling, this $O m d$ promoter region was ligated into a luciferase reporter expression vector (pOmd-luc), and luciferase activity was observed in BMP2-treated C2C12 cells (Fig. 2C). Transient transfection of these cells with pOmd-luc and co-transfection with Smad1 (DVD) and Smad4 expression plasmids resulted in a significant increase in luciferase activity (Fig. 2D). However, co-transfection with Smad1 (DVD) and Smad4 expression plasmids did not alter pOmd-luc activity in MC3T3-E1 cells (data not shown).

Knockdown of $Y 1$ receptor upregulates Omd $m R N A$ expression in MC3T3-E1 cells. Several studies have reported that neuropeptide $\mathrm{Y}$ and its $\mathrm{Y} 1$ receptor are directly involved in osteoblast regulation (24-26). Previously, we demonstrated that knockdown of the Y1 receptor using siRNA promotes osteoblast differentiation (16). Therefore, the present study examined the effects of $\mathrm{Y} 1$ receptor inhibition on $\mathrm{Omd}$ mRNA expression. Following transfection of MC3T3-E1 cells with siRNA for the Y1 receptor, Y1 receptor $(N p y l r)$ mRNA expression level was decreased to undetectable levels, confirming that the siRNA was effective at silencing endogenous Y1 receptor expression (Fig. 3A). Under these conditions, Omd mRNA expression was significantly increased (Fig. 3B), indicating that $O m d$ expression is Y1 receptor dependent and that the NPY signaling pathway regulates Omd expression in MC3T3-E1 cells.

Effects of overexpression or knockdown of Omd on viability and apoptosis in MC3T3-E1 cells. To determine the biological 
A

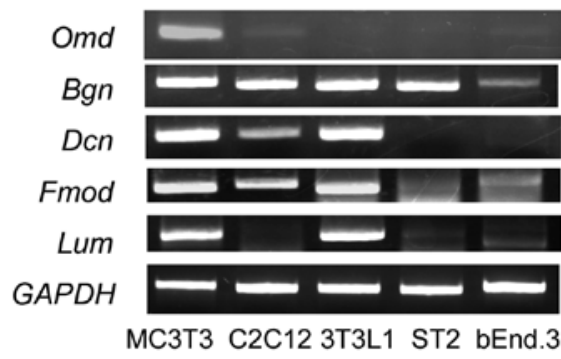

C

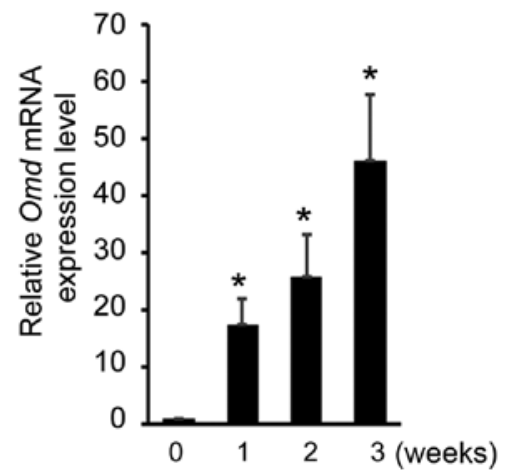

B
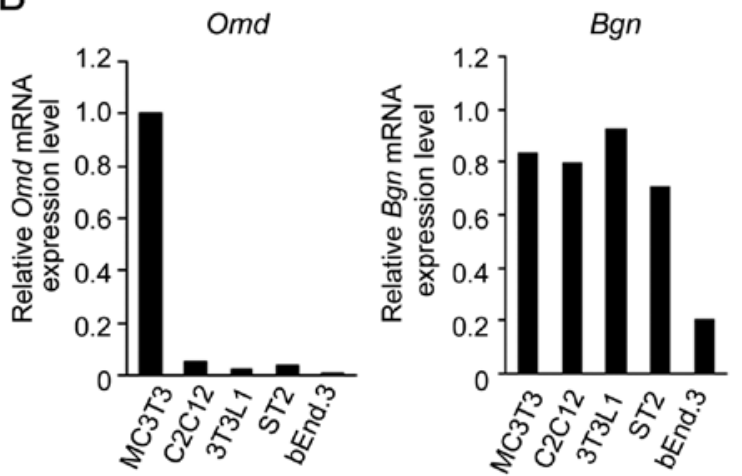

D

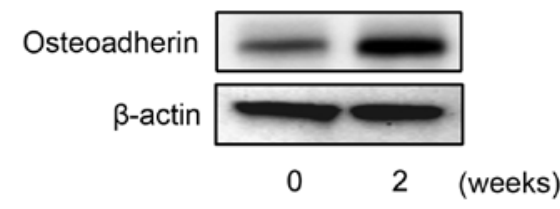

Figure 1. SLRP mRNA expression levels under various culture conditions. (A) Total RNA was extracted from MC3T3-E1, 3T3-L1, C2C12, ST2 and bEnd.3 cells, and mRNA expression levels of the SLRPs Omd, Dcn, Bgn, Fmod and Lum were determined using RT-semi-qPCR analyses. Equal loading of cDNA samples was confirmed by amplification of $G A P D H$ cDNA. The presented data are representative of three experiments with similar results. (B) mRNA expression levels of $O m d$ and Bgn normalized to GAPDH. (C) Confluent MC3T3-E1 cells were incubated in differentiation media for 1-3 weeks. Total RNA was extracted from cells and $O m d$ mRNA expression was determined using RT-qPCR. mRNA expression levels were normalized to those of $G A P D H$, and are presented as fold changes relative to the expression levels in control cells. (D) Confluent MC3T3-E1 cells were incubated in differentiation media for 2 weeks. Total protein was extracted from MC3T3-E1 cells and the levels of Omd protein were determined by western blot analysis. $\beta$-actin was used as an endogenous control. Data are presented as the mean \pm standard deviation of separate experiments performed in triplicate. * $\mathrm{P}<0.05$ vs. week 0 . SLRP, small leucine-rich proteoglycan; Omd, osteoadherin; Dcn, decorin; Bgn, biglycan; Fmod, fibromodulin; Lum, lumican; RT, reverse transcription; qPCR, quantitative PCR; cDNA, complementary DNA.

relevance of $O m d$ regulation in osteoblast differentiation, Omd was significantly overexpressed in MC3T3-E1 cells by transfecting cells with the pOmd expression plasmid (Fig. 4A). Western blot analysis demonstrated that osteoadherin protein was overexpressed in pOmd-transfected MC3T3-E1 cells (Fig. 4B). Viability was significantly increased in these cells compared with those transfected with control plasmid (Fig. 4C). Caspase 3/7 activity, which is associated with apoptosis (15), was also significantly decreased following transfection with pOmd (Fig. 4D), which further indicates that $\mathrm{Omd}$ is involved in the regulation of apoptosis in MC3T3-E1 cells.

In further experiments, the effects of Omd-knockdown was examined using RNA interference in MC3T3-E1 cells. Following transfection with $O m d$ siRNA, Omd mRNA expression was significantly decreased (Fig. 4E). As presented in Fig. 4F, cells transfected with $O m d$ siRNA exhibited a reduction in osteoadherin expression compared with cells transfected with siCont. Viable cell numbers were decreased compared with those in control siRNA-transfected cells, although statistical significance between control siRNA- and Omd siRNA-transfected cells was not confirmed (Fig. 4G). By contrast, caspase 3/7 activity was significantly increased following exposure to $\mathrm{Omd}$ siRNA (Fig. $4 \mathrm{H}$ ). These results indicate that cell survival/death is $O m d$-dependent and that osteoadherin may regulate cell growth and apoptosis in osteoblast cells.

Effects of Omd overexpression on the mRNA expression of specific osteoblast differentiation genes in MC3T3-E1 cells. To investigate the effects of osteoadherin in osteoblast differentiation, the present study examined the effects on genes with characterized roles in osteoblast differentiation. Following transfection with $O m d$ siRNA, Alp mRNA expression level and ALP activities were not affected in MC3T3-E1 cells (data not shown). Although Alp and Bglap mRNA expression levels were not significantly altered by $O m d$ overexpression, $C c n 2$ expression was significantly reduced (Fig. 5A). However, as ALP activity was not affected by $O m d$ overexpression on day 14 (Fig. 5B), it can be concluded that osteoblast-related gene expression is not dependent on $\mathrm{Omd}$ in MC3T3-E1 cells.

\section{Discussion}

SLRP family members are secreted into the ECM after synthesis by the cells of connective tissues. These proteoglycans have core proteins with LRRs and are important 
A

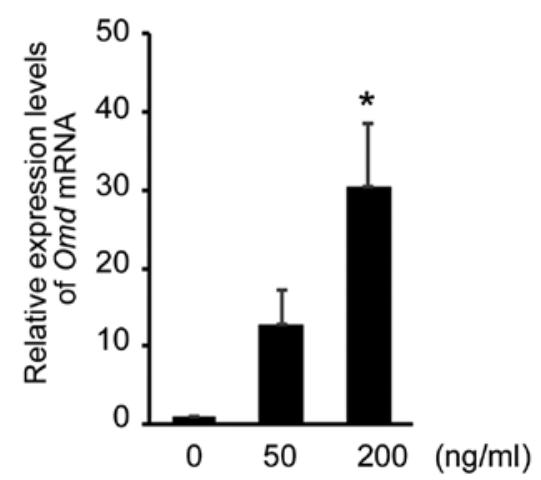

B

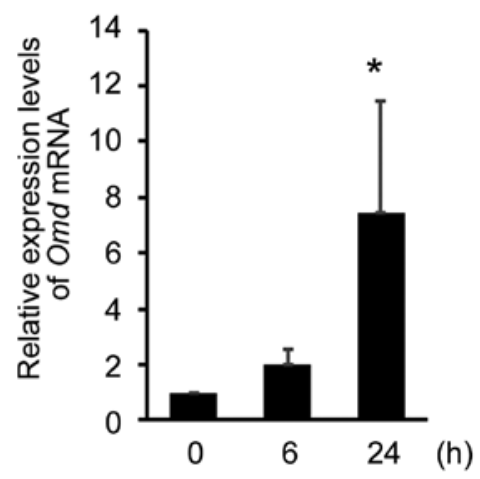

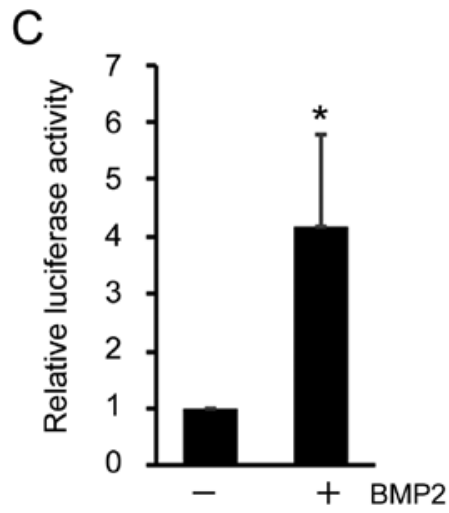

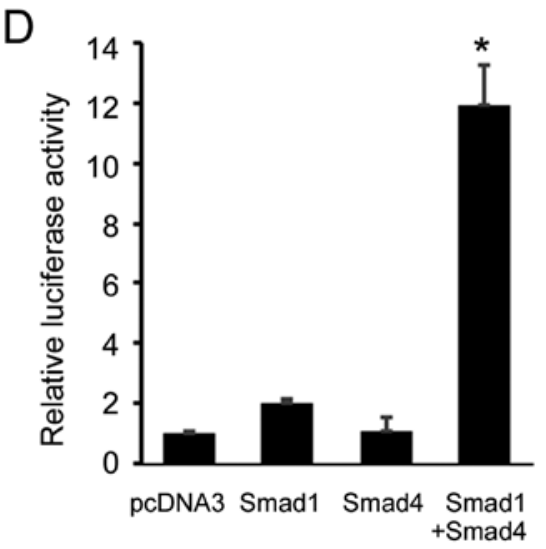

Figure 2. BMP2 signaling induces $O m d$ expression and $O m d$ promoter activity in $\mathrm{C} 2 \mathrm{C} 12$ cells. (A) $\mathrm{C} 2 \mathrm{C} 12$ cells were seeded into culture dishes at $1.5 \times 10^{6}$ cells $/ \mathrm{cm}^{2}$. On day 1 of culture, cells were cultured in media containing indicated concentrations of BMP2 or vehicle (0), and total RNA was extracted from cells after 2 days. ${ }^{*} \mathrm{P}<0.05 \mathrm{vs} .0 \mathrm{ng} / \mathrm{ml}$. (B) C2C12 cells were seeded into $100-\mathrm{mm}$ cell culture dishes and, after $24 \mathrm{~h}$, were cultured in fresh media containing $200 \mathrm{ng} / \mathrm{ml}$ BMP2 for the indicated times. Omd mRNA expression was determined using reverse transcription-quantitative PCR. "P $<0.05 \mathrm{vs.} 0 \mathrm{~h}$. (C) $\mathrm{C} 2 \mathrm{C} 12$ cells were transiently transfected with the pOmd-luc reporter plasmid in 24-well plates. Subsequently, $200 \mathrm{ng} / \mathrm{ml}$ BMP2 (+) or vehicle (-) was added and cells were cultured for a further $24 \mathrm{~h}$ prior to determinations of luciferase activity. (D) C2C12 cells were transiently co-transfected in 24-well plates with the reporter plasmid pOmd-luc and expression constructs for Smad1 or Smad4, or an empty expression plasmid (pcDNA3). Luciferase activities were determined after $48 \mathrm{~h}$. Normalized luciferase activities are presented as relative ratios. ${ }^{*} \mathrm{P}<0.05 \mathrm{vs}$. pcDNA3. Data are presented as the mean \pm standard deviation from separate experiments performed in triplicate. BMP, bone morphogenetic protein; Omd, osteoadherin.
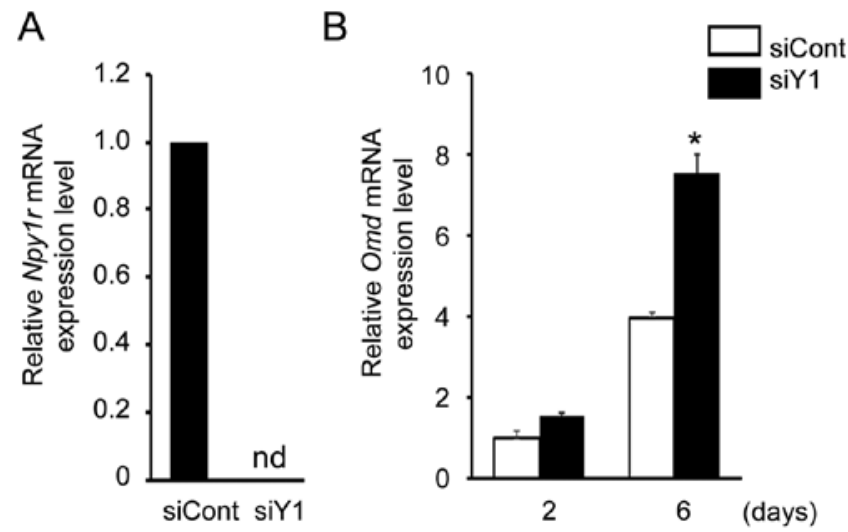

Figure 3. Knockdown of the Y1 receptor upregulates $O m d$ mRNA expression in MC3T3-E1 cells. (A) MC3T3-E1 cells were plated at $1.7 \times 10^{4}$ cells $/ \mathrm{cm}^{2}$ and were cultured for $24 \mathrm{~h}$. Cells were then transfected with siY1 or siCont (both at $5 \mathrm{nM}$ ) and were incubated again for 2-6 days. Total RNA was extracted, and (A) after 2 days $N p y 1 r$, and (B) after 2 and 6 days $O m d$ mRNA expression levels were determined using reverse transcription-quantitative PCR analyses. Expression levels were normalized to those of GAPDH and fold changes were calculated relative to the control. Data are presented as the mean \pm standard deviation of separate experiments performed in triplicate. "P<0.05. siY1, Y1 receptor small interfering RNA; siCont, scramble small interfering RNA; Omd, osteoadherin; Npylr, Y1 receptor.

structural components of the ECM. Accordingly, numerous SLRPs have been reported to bind multiple ECM constituents, particularly fibrillar collagens, and thereby stabilize tissue frameworks $(7,8)$. SLRPs include five classes of structurally related proteoglycans. Among them, osteoadherin, fibromodulin, keratocan and lumican are class II SLRPs (2), 
A

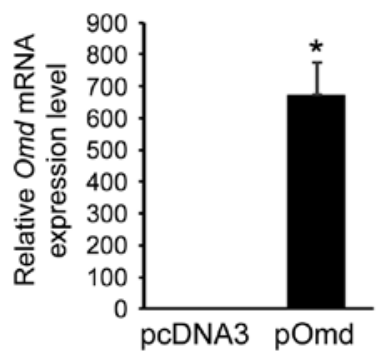

D

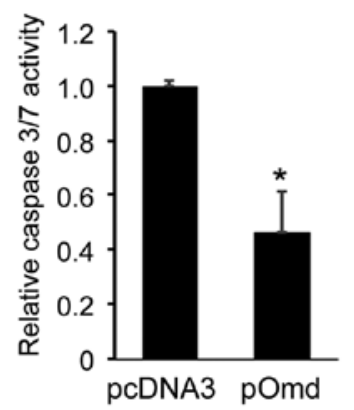

B

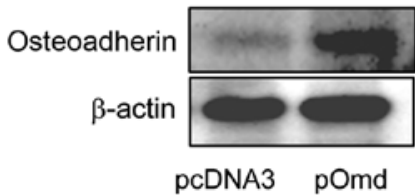

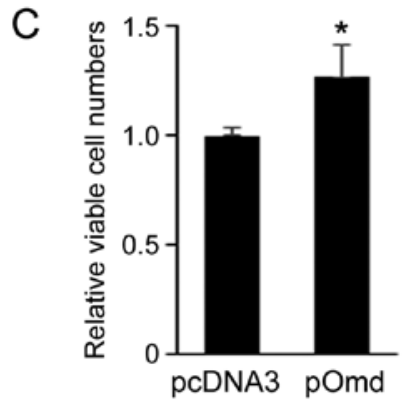

$\mathrm{F}$
E

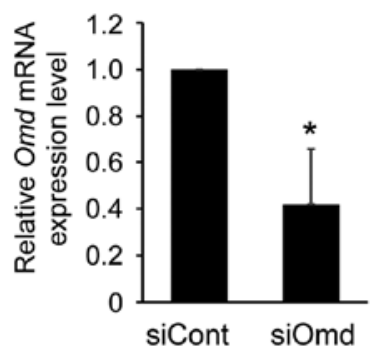

Osteoadherin

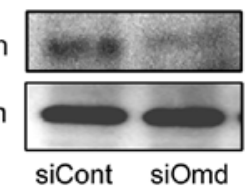

G

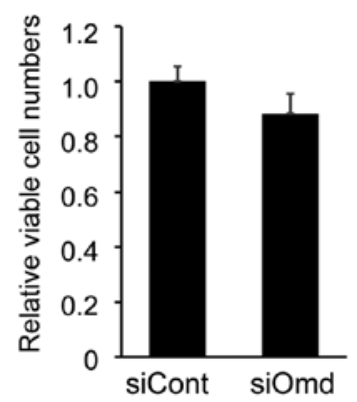

$\mathrm{H}$

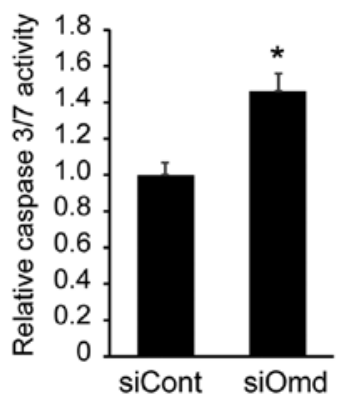

Figure 4. Effects of overexpression and knockdown of Omd on cell proliferation and apoptosis in MC3T3-E1 cells. MC3T3-E1 cells were plated at $1.0 \times 10^{5}$ cells $/ \mathrm{cm}^{2}$. Following culture for $24 \mathrm{~h}$, cells were transfected with pOmd or pcDNA3. (A) Total RNA was extracted after 2 days and Omd mRNA expression levels were determined using RT-qPCR. (B) Total protein was extracted from MC3T3-E1 cells and the levels of osteoadherin protein were determined by western blot analysis. (C) Viable cell numbers were quantified. (D) The activity of caspase 3/7 was measured. (E) MC3T3-E1 cells were plated at $1.7 \times 10^{4}$ cells $/ \mathrm{cm}^{2}$. Following culture for $24 \mathrm{~h}$, cells were transfected with siOmd or siCont (both at $5 \mathrm{nM}$ ). Total RNA was extracted after 1 day and $O m d$ mRNA expression levels were determined using RT-qPCR. (F) Total protein was extracted from MC3T3-E1 cells and the levels of osteoadherin protein were determined by western blot analysis. (G) Viable cell numbers were quantified. (H) The activity of caspase 3/7 was measured. (A and E) Expression levels were normalized to those of GAPDH and are presented as fold changes relative to the control. (B and F) $\beta$-actin was used as an endogenous control. Fold changes in viable cell numbers and activities were calculated on the basis of those in control cells (those transfected with siCont or pcDNA3). Data are presented as the mean \pm standard deviation of separate experiments performed in triplicate. ${ }^{*} \mathrm{P}<0.05$ vs. pcDNA3 or siCont. Omd, osteoadherin; siOmd, Omd small interfering RNA; siCont, scramble small interfering RNA; pOmd, Omd overexpression plasmid; RT-qPCR, reverse transcription-quantitative PCR.

A

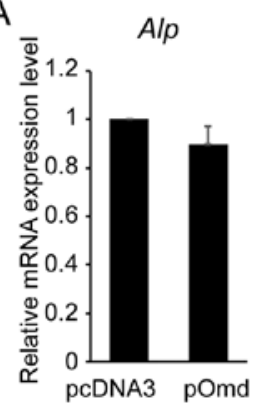

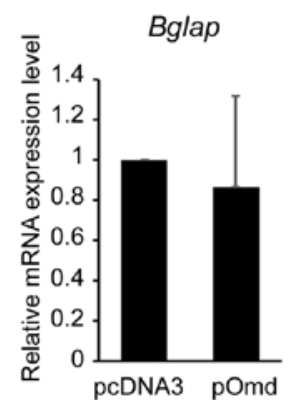

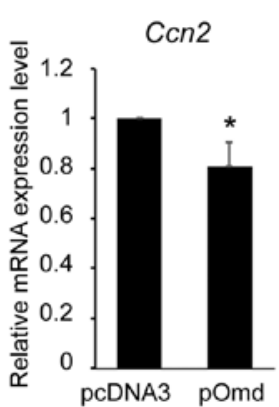

B

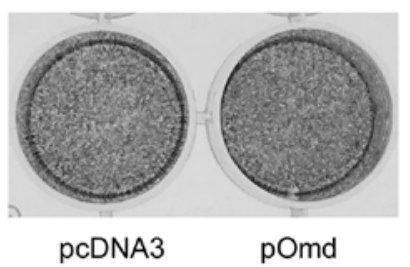

Figure 5. Effects of Omd overexpression on the mRNA expression levels of genes related to osteoblast differentiation in MC3T3-E1 cells. MC3T3-E1 cells were plated at $1.7 \times 10^{4}$ cells $/ \mathrm{cm}^{2}$ and were cultured. After $24 \mathrm{~h}$, cells were transfected with pOmd or pcDNA3 plasmids and were incubated. (A) Total RNA was then extracted after 2 days and Alp, Bglap and Ccn2 mRNA expression levels were determined using reverse transcription-quantitative PCR. Fold changes in mRNA expression levels were calculated relative to expression levels in control cells transfected with pcDNA3. Data are presented as the mean \pm standard deviation from separate experiments performed in triplicate. " $\mathrm{P}<0.05$ vs. pcDNA3. (B) After transfection with pOmd or pcDNA3, cells were cultured for 14 days and ALP activities were investigated using staining analyses. Bglap, osteocalcin; Alp, alkaline phosphatase; Ccn2, CCN family 2; Omd, osteoadherin; pOmd, Omd overexpression plasmid. 
and osteoadherin has highly restricted expression in cells of mineralized tissues $(4,6)$. In the present study, BMP2 enhanced Omd expression in $\mathrm{C} 2 \mathrm{C} 12$ cells. Y1R inhibition also enhanced Omd expression, suggesting specific functions of osteoadherin in bone homeostasis and osteoblast differentiation. The class I SLPRs biglycan and decorin are also expressed in osteoblasts and are present in bone $\operatorname{ECM}(2,27)$, and their affinity for bone apatite has been demonstrated by chromatographic purification with hydroxyapatite columns (28). These observations suggest significant roles of these SLPRs in the regulation of mineralization (28). Osteoadherin also interacts with calcium phosphate mineral (29), suggesting that osteoadherin contributes to biomineralization and the control of mineral crystal nucleation, growth, and maturation.

Althoughfew previous reports show regulation of $O m d$ expression by growth factors and signaling molecules, Rehn et al (30) reported that transforming growth factor- $\beta$ (TGF- $\beta$ ) downregulates $O m d$ expression in osteoblasts. The present study demonstrated that BMP2 enhances Omd gene expression and activates the Omd gene promoter. Upon activation by BMP2, Smad1/Smad 4 complexes regulate the transcription of various target genes, and among these $I d 1, T l x-2$ and Mix.2 reportedly respond to BMP signaling via the Smad-binding motifs on their promoters $(23,31,32)$. Several DNA binding motifs for Smads, including GTCT, have been identified (33). The present analyses indicate the presence of transcriptional machinery that is sensitive to BMP2 signaling and regulates transcription through interactions with the Omd promoter.

SLRPs have multiple complex roles. Class II SLRPs contain charged KS chains in their LRRs and $\mathrm{N}$ termini with multiple sulfated tyrosine residues that contribute to the anionic properties of these proteoglycans. The GAG $\mathrm{KS}$ is reportedly present in several tissues, including bone, cartilage, reproductive and neural tissues $(1,2)$. KS binds the growth factors fibroblast growth factor 2 , TGF- $\beta$ and sonic hedgehog with high affinity $(34,35)$, suggesting roles of KS proteoglycans in the regulation of growth factor activities and morphogen gradient formation. Decorin has been characterized as a proteoglycan with roles in the control of cell growth (2), as indicated by binding and inhibition of TGF- $\beta$. Biglycan also interacts with TGF- $\beta$ and modulates its activities (2). In addition, decorin was reported to interact with epidermal growth factor receptor and vascular endothelial growth factor receptor, and consequently regulate the cell cycle $(2,9,36)$. The KS of osteoadherin interacts with fibronectin to promote osteoblast attachment in vitro (3). Furthermore, the tyrosine sulfate-rich domains of osteoadherin have been shown to bind motifs of basic clusters in a variety of heparin-binding proteins, including some with bioactive properties (10). These studies suggest that osteoadherin binds various growth factors, growth factor receptors and matrix proteins that regulate apoptosis and cell growth via cell cycle- and proliferation-related mechanisms.

CCN2, which is widely considered a connective tissue growth factor, is a cysteine-rich ECM protein that acts as an anabolic growth factor to regulate cell functions $(37,38)$. Ccn2 mRNA expression also induces apoptosis in human aortic smooth muscle cells (39), breast cancer cells (40) and mouse osteocytes (41) by down-regulating anti-apoptotic genes. In accordance with these observations, Omd overexpression reduced Ccn2 expression in MC3T3-E1 cells in the present study, suggesting an apoptotic mechanism involving CCN2 that is regulated by osteoadherin expression in osteoblasts.

In conclusion, the present study, to the best of our knowledge, is the first to show that osteoadherin regulates apoptosis and proliferation in osteoblast cells, and thus established a molecular relationship between osteoblast-derived SLRP osteoadherin and caspase 3/7 activity in osteoblasts. However, the mechanism of regulation on caspase $3 / 7$ activity by osteoadherin remains unclear. Further investigations are required to identify molecular mechanisms of osteoadherin and associated signaling molecules.

\section{Acknowledgements}

Not applicable.

\section{Funding}

The present study was supported in part by a research grant from the Japan Society for the Promotion of Science Grants-in-aid for Scientific Research (grant no. 17K1163707).

\section{Availability of data and materials}

The datasets generated and/or analyzed during the present study are available from the corresponding author on reasonable request.

\section{Authors' contributions}

EH and MT conceived and designed the experiments. EH performed the experiments. $\mathrm{EH}$ and TF analyzed the data. $\mathrm{EH}$ and MT wrote the manuscript. All authors read and approved the final manuscript for publication.

\section{Ethics approval and consent to participate}

Not applicable.

\section{Patient consent for publication}

Not applicable.

\section{Competing interests}

The authors declare that they have no competing interests.

\section{References}

1. Heinegård D: Fell-Muir Lecture: Proteoglycans and more-from molecules to biology. Int J Exp Pathol 90: 575-586, 2009.

2. Iozzo RV and Schaefer L: Proteoglycan form and function: A comprehensive nomenclature of proteoglycans. Matrix Biol 42: $11-55,2015$

3. Wendel M, Sommarin Y and Heinegård D: Bone matrix proteins: Isolation and characterization of a novel cell-binding keratan sulfate proteoglycan (osteoadherin) from bovine bone. J Cell Biol 141: 839-847, 1998.

4. Sommarin Y, Wendel M, Shen Z, Hellman U and Heinegârd D: Osteoadherin, a cell-binding keratan sulfate proteoglycan in bone, belongs to the family of leucine-rich repeat proteins of the extracellular matrix. J Biol Chem 273: 16723-16729, 1998. 
5. Buchaille R, Couble ML, Magloire H and Bleicher F: Expression of the small leucine-rich proteoglycan osteoadherin/osteomodulin in human dental pulp and developing rat teeth. Bone 27: 265-270, 2000

6. Nikdin H, Olsson ML, Hultenby K and Sugars RV: Osteoadherin accumulates in the predentin towards the mineralization front in the developing tooth. PLoS One 7: e31525, 2012.

7. Sjöberg AP, Manderson GA, Mörgelin M, Day AJ, Heinegård D and Blom AM: Short leucine-rich glycoproteins of the extracellular matrix display diverse patterns of complement interaction and activation. Mol Immunol 46: 830-839, 2009

8. Kalamajski S and Oldberg A: The role of small leucine-rich proteoglycans in collagen fibrillogenesis. Matrix Biol 29: $248-253,2010$

9. Schaefer L and Iozzo RV: Biological functions of the small leucine-rich proteoglycans: From genetics to signal transduction. J Biol Chem 283: 21305-21309, 2008.

10. Tillgren V, Onnerfjord P, Haglund L and Heinegård D: The tyrosine sulfate-rich domains of the LRR proteins fibromodulin and osteoadherin bind motifs of basic clusters in a variety of heparin-binding proteins, including bioactive factors. J Biol Chem 284: 28543-28553, 2009.

11. Rehn AP, Cerny R, Sugars RV, Kaukua N and Wendel M: Osteoadherin is upregulated by mature osteoblasts and enhances their in vitro differentiation and mineralization. Calcif Tissue Int 82: 454-464, 2008.

12. Canalis E, Economides AN and Gazzerro E: Bone morphogenetic proteins, their antagonists, and the skeleton. Endocr Rev 24 218-235, 2003

13. Chen G, Deng $C$ and Li YP: TGF- $\beta$ and BMP signaling in osteoblast differentiation and bone formation. Int J Biol Sci 8 $272-288,2012$

14. Katagiri $\mathrm{T}$ and Tsukamoto $\mathrm{S}$ : The unique activity of bone morphogenetic proteins in bone: A critical role of the Smad signaling pathway. Biol Chem 394: 703-714, 2013

15. Van Opdenbosch N and Lamkanfi M: Caspases in cell death, inflammation, and disease. Immunity 50: 1352-1364, 2019.

16. Yahara M, Tei K and Tamura M: Inhibition of neuropeptide $\mathrm{Y} Y 1$ receptor induces osteoblast differentiation in MC3T3-E1 cells. Mol Med Rep 16: 2779-2784, 2017.

17. Tamura M, Sato MM and Nashimoto M: Regulation of CXCL12 expression by canonical Wnt signaling in bone marrow stroma cells. Int J Biochem Cell Biol 43: 760-767, 2011.

18. Montesano R, Pepper MS, Möhle-Steinlein U, Risau W, Wagner EF and Orci L: Increased proteolytic activity is responsible for the aberrant morphogenetic behavior of endothelial cells expressing the middle T oncogene. Cell 62: 435-445, 1990.

19. Tsuji-Tamura $\mathrm{K}$ and Ogawa M: Dual inhibition of mTORC1 and mTORC2 perturbs cytoskeletal organization and impairs endothelial cell elongation. Biochem Biophys Res Commun 497: 326-331, 2018

20. Nakashima A, Katagiri T and Tamura M: Cross-talk between Wnt and bone morphogenetic protein 2 (BMP-2) signaling in differentiation pathway of C2C12 myoblasts. J Biol Chem 280 37660-37668, 2005.

21. Iizuka S, Oridate N, Nashimoto M, Fukuda S and Tamura M Growth inhibition of head and neck squamous cell carcinoma cells by sgRNA targeting the cyclin D1 mRNA based on TRUE gene silencing. PLoS One 9: e114121, 2014.

22. Livak KJ and Schmittgen TD: Analysis of relative gene expression data using real-time quantitative PCR and the 2(-Delta Delta C(T)) method. Methods 25: 402-408, 2001
23. Nojima J, Kanomata K, Takada Y, Fukuda T, Kokabu S, Ohte S, Takada T, Tsukui T, Yamamoto TS, Sasanuma H, et al: Dual roles of smad proteins in the conversion from myoblasts to osteoblastic cells by bone morphogenetic proteins. J Biol Chem 285: 15577-15586, 2010.

24. Teixeira L, Sousa DM, Nunes AF, Sousa MM, Herzog H and Lamghari M: NPY revealed as a critical modulator of osteoblast function in vitro: New insights into the role of Y1 and Y2 receptors. J Cell Biochem 107: 908-916, 2009.

25. Khor EC and Baldock P: The NPY system and its neural and neuroendocrine regulation of bone. Curr Osteoporos Rep 10 $160-168,2012$

26. Kurebayashi N, Sato M, Fujisawa T, Fukushima K and Tamura M: Regulation of neuropeptide Y Y1 receptor expression by bone morphogenetic protein 2 in $\mathrm{C} 2 \mathrm{C} 12$ myoblasts. Biochem Biophys Res Commun 439: 506-510, 2013.

27. Nikitovic D, Aggelidakis J, Young MF, Iozzo RV, Karamanos NK and Tzanakakis GN: The biology of small leucine-rich proteoglycans in bone pathophysiology. J Biol Chem 287: 33926-33933, 2012

28. Boskey AL, Spevak L, Doty SB and Rosenberg L: Effects of bone CS-proteoglycans, DS-decorin, and DS-biglycan on hydroxyapatite formation in a gelatin gel. Calcif Tissue Int 61: 298-305, 1997.

29. Zhou HY: Proteomic analysis of hydroxyapatite interaction proteins in bone. Ann N Y Acad Sci 1116: 323-326, 2007.

30. Rehn AP, Chalk AM and Wendel M: Differential regulation of osteoadherin (OSAD) by TGF-beta1 and BMP-2. Biochem Biophys Res Commun 349: 1057-1064, 2006.

31. Tang SJ, Hoodless PA, Lu Z, Breitman ML, McInnes RR, Wrana JL and Buchwald M: The Tlx-2 homeobox gene is a downstream target of BMP signalling and is required for mouse mesoderm development. Development 125: 1877-1887, 1998.

32. Dennler S, Itoh S, Vivien D, ten Dijke P, Huet S and Gauthier JM: Direct binding of Smad3 and Smad4 to critical TGF beta-inducible elements in the promoter of human plasminogen activator inhibitor-type 1 gene. EMBO J 17: 3091-3100, 1998

33. Zawel L, Dai JL, Buckhaults P, Zhou S, Kinzler KW, Vogelstein B and Kern SE: Human Smad3 and Smad4 are sequence-specific transcription activators. Mol Cell 1: 611-617, 1998.

34. Schaefer L and Schaefer RM: Proteoglycans: From structural compounds to signaling molecules. Cell Tissue Res 339: 237-246, 2010.

35. Weyers A, Yang B, Solakyildirim K, Yee V, Li L, Zhang F and Linhardt RJ: Isolation of bovine corneal keratan sulfate and its growth factor and morphogen binding. FEBS J 280: 2285-2293, 2013.

36. Buraschi S, Neill T, Goyal A, Poluzzi C, Smythies J, Owens RT, Schaefer L, Torres A and Iozzo RV: Decorin causes autophagy in endothelial cells via Peg3. Proc Natl Acad Sci USA 110: E2582-E2591, 2013.

37. Chen CC and Lau LF: Functions and mechanisms of action of CCN matricellular proteins. Int J Biochem Cell Biol 41: 771-783, 2009.

38. Kubota S and Takigawa M: Cellular and molecular actions of CCN2/CTGF and its role under physiological and pathological conditions. Clin Sci (Lond) 128: 181-196, 2015.

39. Hishikawa K, Oemar BS, Tanner FC, Nakaki T, Fujii T and Lüscher TF: Overexpression of connective tissue growth factor gene induces apoptosis in human aortic smooth muscle cells. Circulation 100: 2108-2112, 1999.

40. Hishikawa K, Oemar BS, Tanner FC, Nakaki T, Lüscher TF and Fujii T: Connective tissue growth factor induces apoptosis in human breast cancer cell line MCF-7. J Biol Chem 274: 37461-37466, 1999.

41. Sakai Y, Balam TA, Kuroda S, Tamamura N, Fukunaga T, Takigawa $\mathrm{M}$ and Takano-Yamamoto T: CTGF and apoptosis in mouse osteocytes induced by tooth movement. J Dent Res 88: $345-350,2009$. 